\title{
Efficacy of Olyset long-lasting bednets to control transmission of cutaneous leishmaniasis in Iran
}

M.M. Emami, ${ }^{1}$ M. Yazdi ${ }^{2}$ and P. Guillet ${ }^{3}$

$$
\begin{aligned}
& \text { كفاءة الناموسيات المشربة بالأوليست الصامدة لأمد طويل في مكافحة سراية داء الليشمانيات الجلدي في } \\
& \text { إيران } \\
& \text { حممد متولي إمامي، محبوبة يزدي، بـ. غويليت }
\end{aligned}
$$

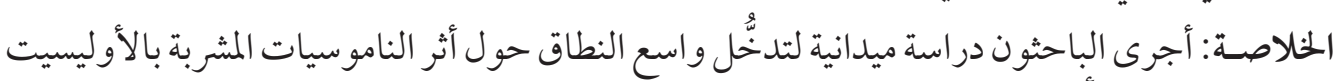

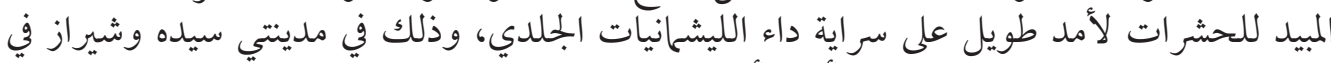

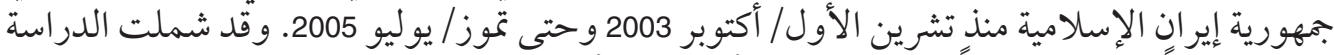

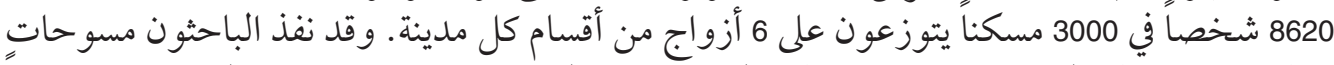

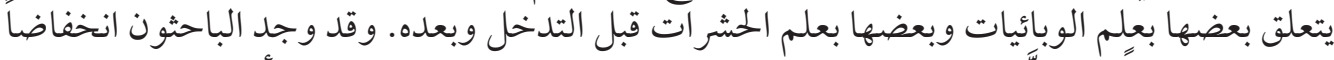

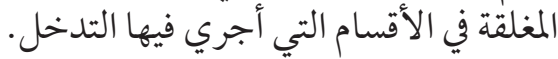

ABSTRACT A large-scale intervention field trial of the effect of Olyset ${ }^{\circledR}$ long-lasting insecticide-treated bednets on transmission of cutaneous leishmaniasis was carried out in 2 cities in the Islamic Republic of Iran from October 2003 to July 2005. We enrolled 8620 individuals in 3000 households in 6 pairs of sectors in each city. Epidemiological and entomological surveys were carried out pre- and post-intervention. In both cities a statistically significant reduction was found in the incidence of new cases in intervention sectors who received bednets compared with control areas. Entomological surveys showed a reduction in numbers of female Phlebotomus sergenti captured indoors in intervention sectors.

Efficacité des moustiquaires Olyset à longue durée d'action dans la lutte contre la transmission de la leishmaniose cutanée en Iran

RÉSUMÉ Un essai pratique à grande échelle relatif aux effets des moustiquaires imprégnées d'insecticide à longue durée d'action Olyset ${ }^{\circledR}$ sur la transmission de la leishmaniose cutanée a été réalisé dans deux villes de la République islamique d'Iran d'octobre 2003 à juillet 2005 . Nous avons recruté 8620 sujets appartenant à 3000 foyers rattachés à 6 paires de secteurs dans chaque ville. Des études épidémiologiques et entomologiques ont été réalisées avant et après l'intervention. Dans les deux villes, une réduction statistiquement significative de l'incidence des nouveaux cas a été observée dans les secteurs d'intervention qui avaient reçu des moustiquaires, par rapport aux zones témoins. Les études entomologiques ont montré une diminution du nombre de femelles de Phlebotomus sergenti capturées à l'intérieur des habitations situées dans les secteurs d'intervention.

'Sepahan Green-Thou Plant Pathology and Medical Entomology Centre, Isfahan, Islamic Republic of Iran (Correspondence to M.M. Emami: motovaliema@yahoo.com).

${ }^{2}$ Kerman University of Medical Sciences, Kerman, Islamic Republic of Iran.

${ }^{3}$ Communicable Diseases Control, Prevention and Eradication, World Health Organization, Geneva, Switzerland. Received: 04/11/06; accepted: 02/05/07

المجلة الصحية لشرق المتوسط، منظمة الصحة العالمية، المجلد الخامس عشر، العدد 0، 9 ب. 


\section{Introduction}

The leishmaniases are parasitic diseases with a wide range of clinical symptoms. Leishmaniasis currently threatens 350 million people in 88 countries around the world, with 1-1.5 million new cases of cutaneous leishmaniasis (CL) reported annually [1].

Both zoonotic and anthroponotic CL are found in scattered foci in various parts of the Islamic Republic of Iran. In recent years, due to the increase in population, there have been increases in areas where leishmaniasis was previously rare, on the southern slopes of the Zagros mountains and in Darab district and Fars province [2], and new foci have been found in central parts of the country, such as Sedeh in Isfahan province [unpublished report, Isfahan University of Medical Sciences]. The sandfly Phlebotomus (Paraphlebotomus) sergenti is the predominant vector in these areas and constitutes $82 \%$ of the total sandflies captured indoors.

Use of insecticide-treated bednets (ITNs) for protection against mosquito bites at night is a highly effective and cost-effective intervention against vector-borne diseases such as malaria $[3,4]$. A case--control study in Nepal showed that people using untreated bednets were $70 \%$ less likely to develop viseral leishmaniasis than people without nets [5]. Protection provided by wide-mesh bednets is enhanced by treating them with pyrethroid, reducing sandfly biting rates by $64 \%-100 \%[6,7]$. There is also evidence from Colombia that sandfly bites are not diverted to people sleeping outside ITNs; unprotected people in the same room as someone sleeping under a deltamethrintreated net received $42 \%$ fewer sandfly bites than people in houses without nets [6].

Long-lasting ITNs are treated at factory level with insecticide, either incorporated into or coated around the fibres; they are re- sistant to multiple washes and have biological activity that lasts as long as the net itself (3-4 years for polyester nets) [8]. Olyset Net ${ }^{\circledR}$ is a polyethylene net impregnated with $2 \%$ permethrin and is effective against malaria vectors for at least 5 years. Its use is recommended by the World Health Organization [8]. Olyset ITNs showed deterrence against Anopheles gambiae in Cote d'Ivoire (44\% reduction in mosquito population with new nets, $20 \%$ with dirty bednets, none with washed nets) [9].

In the past, control measures carried out mainly in the Shiraz and Isfahan area of the Islamic Republic of Iran failed to permanently decrease the number of cases of anthroponotic CL (unpublished data). We therefore carried out a study in urban areas of 2 provinces with intense transmission of CL. We assessed any reduction in the abundance of sandflies indoors and of clinical cases of CL in areas where households were protected by Olyset ITNs compared with areas with households not using ITNs.

\section{Methods}

\section{Study area}

The data reported for the intervention and post-intervention periods were collected between April 2004 and July 2005 in 2 cities in the Islamic Republic of Iran: Sedeh and Shiraz. Data from October 2003 to April 2004 (pre-intervention survey) were obtained from previous research by Isfahan University of Medical Sciences [10].

Sedeh city $\left(32^{\circ} 41^{\prime} \mathrm{N}, 51^{\circ} 31^{\prime} \mathrm{E}\right)$ is located in Isfahan province in the south-west of the country, $12 \mathrm{~km}$ from Isfahan city. It is $1602 \mathrm{~m}$ above sea level and the climate is warm and dry with 4 distinct seasons. The mean annual rainfall over the previous 38 years was $117 \mathrm{~mm}$ and relative humidity $44 \%$. The mean monthly temperature 
ranges from $48{ }^{\circ} \mathrm{C}$ to $5^{\circ} \mathrm{C}$. Sedeh has a population of around 300000 . In recent years immigration has played an important role in the population increase. There are 1500 stone-cutting factories for buildings in this industrial city.

Shiraz city $\left(29^{\circ} 38^{\prime} \mathrm{N}, 52^{\circ} 34^{\prime} \mathrm{E}\right)$ is located in the south-west of the country with 1050000 inhabitants and it is the capital of Fars province. It is $1486 \mathrm{~m}$ above sea level in the Zagros mountains and has a moderate climate, with a hot dry summer (JulySeptember), a warm wet autumn (OctoberDecember), a mild winter (January-March) and a warm and rainy spring (April-June). The city supports industries such as cement manufacture, sugar, fertilizers, textile products, wood products, metalwork and rug-making, and is a trading centre for provincial crops such as grapes, citrus fruits, cotton and rice.

In both cities the residential buildings are of traditional design constructed with clay and brick dating back over 25 years. Compounds usually consist of an entrance leading to a courtyard with a lavatory and houses have 3 or more rooms, including a living room, hall, small kitchen, bedroom and bathroom. Animal quarters are sometimes built at the furthest end of the same compound opposite the living area but generally they are built under the living room separated by a cement wall.

\section{Study population}

The populations are mainly subsistence farmers practising animal husbandry, with some working as labourers in nearby factories and offices. The main activities of the women consist of household duties, carpetweaving and crafts. The inhabitants perform various activities, including carpet-making, in the courtyards of their houses in the daytime during summer. They also watch television and sleep in the courtyards us- ing a traditional sleeping sheet on summer nights. In both areas people usually wake up at 04.00-05.00 hours to pray and then sleep until 06.00-07.00 and most of them sleep at $21.00-24.00$ in the courtyard.

\section{Study design}

In each city 6 urban sectors were selected based on the pre-intervention epidemiological survey of disease in the area so that all sectors had a similar size and distribution of disease. The 12 sectors in the 2 cities had a total of 3000 houses and 8620 inhabitants. For each of the 6 pairs we used computer-generated random numbers to allocate 1 sector to receive Olyset ITNs (intervention group) and the other sector to receive no nets (control group). Each sector in a pair was at least $2 \mathrm{~km}$ away from the other.

\section{Data collection}

\section{Pre-intervention}

Between April 2004 and July 2004 the preintervention survey on human infection was conducted in the chosen operational areas. Pre-intervention studies were carried out in 2 population groups: first, the inhabitants of areas in which most active cases of CL were recorded by health centres were examined and forms were completed for each household during a house-to-house visit; second, students in all elementary schools were examined and questioned in the 2 cities [10]. The interviewers examined any scars and ulcers, recording cases that occurred during the 9 months before the interview. Interviewers also checked for new cases reported in the leishmaniasis clinic in the previous 9 months.

We used a questionnaire to gather data on the demographic (age and sex) and socioeconomic (education, occupation, housing conditions) characteristics of the population as well as on people's individual 
past history of CL, the sleeping patterns of families and, according to these patterns, the number of bednets that would be distributed.

\section{Intervention}

ITNs were distributed to 1500 households in the intervention sectors in August 2004. The nets were family sized and therefore 1 net was given to each house with 2-3 persons. The standard Olyset ITN weighs about $750 \mathrm{~g}$ with a surface area $14 \mathrm{~m}^{2}$. The bednets have good ventilation, have no need for retreatment. All the ITNs ordered were white and made by Changzhou Jiusi Fibre Product Co., China.

To ensure correct use of the bednets, 59 training sessions for families in the intervention group were carried out in schools and mosques. Health education messages were disseminated to ensure participants' compliance with the proper use of bednets and that they did not use other methods of preventing sandflies. Also, home visits were made to coordinate the distribution, answer the participants' questions and ensure community acceptability.

Families in the control sectors received no ITNs.

\section{Post-intervention}

To determine the pattern of CL incidence and to compare the control and intervention groups, follow-up questionnaires and examinations were conducted every month between August 2004 and July 2005. All members of the participating households were examined. The presence or absence of CL ulcers was indicated on the forms. The reduction in incidence of new cases was the main indicator.

\section{Entomological survey}

Sandflies were collected once a month from fixed sites indoors (bedrooms, stables, lava- tories, etc.) and from outdoor courtyards using 30 sticky traps (castor oil-coated white papers $20 \mathrm{~cm} \times 30 \mathrm{~cm}$ ) from the beginning to the end of the active season (July-October). For the sampling of exophilic species, 20 light traps were run every month between 18.00 and 06.00 hours in each sampling station.

For species identification sandflies were mounted in Puri medium (Sepahan Green-Thou Plant Pathology and Medical Entomology Centre) and identified after 24 hours using the keys of Theodor and Mesghali [11], before being segregated by sex. The common sandfly in the 2 areas has been previously reported as $P$. sergenti [10].

This study was supported by the ViceDean for Research Affairs of Isfahan University of Medical Sciences and the Sepahan Green-Thou Plant Pathology and Medical Entomology Centre.

\section{Statistical analysis}

The chi-squared test was used to compare between proportions. Comparison between the incidence of CL before and after the intervention was performed using paired Student $t$-tests. Due to the longitudinal nature of the study design the relative risk of using Olyset ITNs in the intervention compared to the control areas and in the intervention areas after the use of ITNs compared to baseline was computed.

\section{Results}

Approximately 15000 persons were interviewed before the intervention, and 7636 were included in the selected study sectors. Following educational sessions and information campaigns in intervention areas, ITN coverage was $100 \%$. The monthly data for CL cases from October 2003 to July 


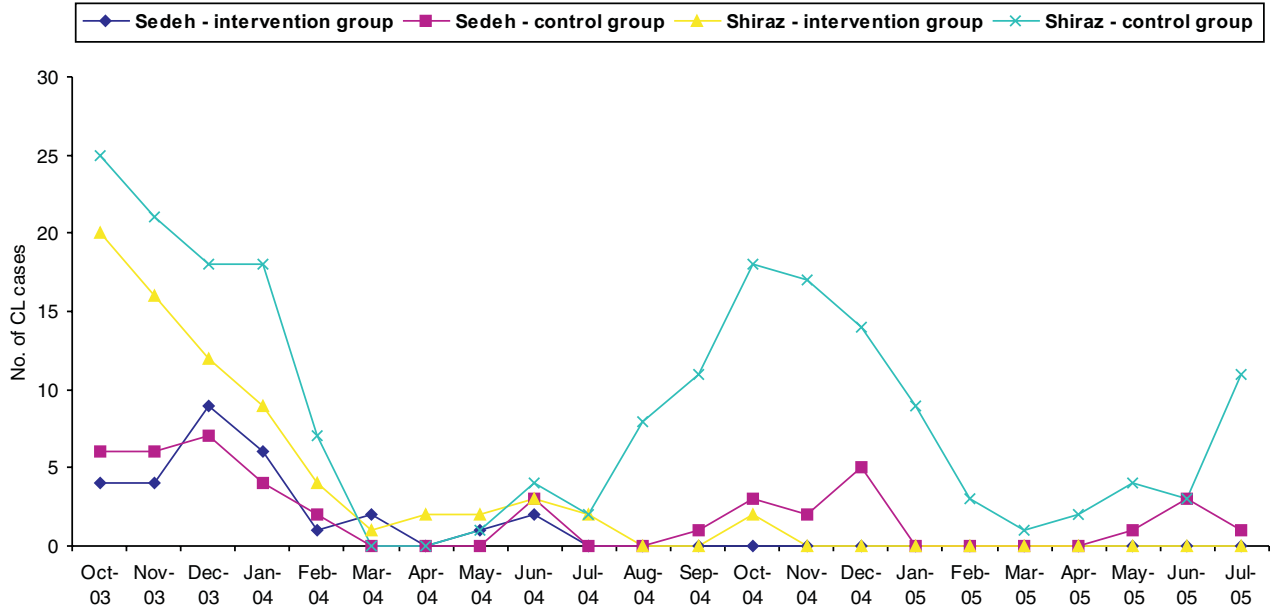

Figure 1 Seasonal fluctuations of number of cases of anthroponotic cutaneous leishmaniasis (CL) in the control and intervention areas in Sedeh and Shiraz cities, October 2003 to July 2005

2005 for the control and intervention sites in the 2 cities is shown in Figure 1.

\section{Sedeh city}

In the 9 months before the intervention (October 2003 to July 2004) there were 29 cases of CL in the intervention group versus 28 in the control group, giving a cumulative estimated incidence of $1.6 \%$ in the intervention sectors and $1.5 \%$ in the control sectors. This difference was not significant (Table 1).
After the intervention (August 2004-July 2005) there were no CL cases in any of the intervention sectors and 16 cases in the control sectors, incidence rates of $0.0 \%$ and $0.9 \%$ respectively (Table 1 ). The difference for CL incidence in intervention sectors before and after the intervention was statistically significant $(P<0.05)$. These results indicate that there was about a $100 \%$ reduction in the intervention area and $58 \%$ decrease in the control area. The relative

Table 1 Cumulative incidence of anthroponotic cutaneous leishmaniasis (CL) estimated from October 2003 to July 2004 (before intervention) and from August 2004 to July 2005 (after intervention) in intervention and control areas of Sedeh and Shiraz cities

\begin{tabular}{|c|c|c|c|c|c|c|}
\hline \multirow[t]{2}{*}{ City/sector } & \multicolumn{3}{|c|}{ Before intervention } & \multicolumn{3}{|c|}{ After intervention } \\
\hline & $\begin{array}{c}\text { No. of people } \\
\text { exposed }\end{array}$ & $\begin{array}{l}\text { No. of new } \\
\text { CL cases }\end{array}$ & $\begin{array}{c}\text { Incidence } \\
\%\end{array}$ & $\begin{array}{c}\text { No. of people } \\
\text { exposed }\end{array}$ & $\begin{array}{l}\text { No. of new } \\
\text { CL cases }\end{array}$ & $\begin{array}{c}\text { Incidence } \\
\%\end{array}$ \\
\hline \multicolumn{7}{|l|}{ Sedeh } \\
\hline Intervention & 1812 & 29 & 1.6 & 1809 & 0 & 0.0 \\
\hline Control & 1820 & 28 & 1.5 & 1820 & 16 & 0.9 \\
\hline \multicolumn{7}{|l|}{ Shiraz } \\
\hline Intervention & 2006 & 71 & 3.5 & 2001 & 2 & 0.1 \\
\hline Control & 1998 & 96 & 4.8 & 1995 & 101 & 5.1 \\
\hline
\end{tabular}

المجلة الصحية لشرق المتوسط، منظمة الصحة العالمية، المجلد الخامس عشر، العدد 0، 9 · 
risk of using Olyset nets in the intervention districts of Sedeh compared with the control ones was 0 and the efficacy was 1 . Therefore Olyset ITNs prevented 100\% of cases of anthroponotic CL in the intervention areas.

In the pre-intervention period, the agent of CL in Sedeh city was confirmed as Leishmania tropica by the analysis of 37 isolates, and the main vector species was identified as $P$. sergenti (56\% prevalence among 2801 sandflies trapped in Sedeh).

In the control sectors the age group 31-40 years had the highest infection rate; the lowest rate was in the age group 11-20 years. The prevalence of CL ulcers differed significantly with sex, prevalence in men being more than twice that in women. More than $63 \%$ of those with active lesions had 1 ulcer, 9.1\% had 2 and the rest had 3 . Five isolates from human indigenous cases in the control area were identified as $L$. tropica.

\section{Shiraz city}

In the 9 months before the intervention there were 71 cases of CL in the intervention group versus 96 in the control group, giving a cumulative estimated incidence of $3.5 \%$ in the intervention sectors and $4.8 \%$ in the control sectors (Table 1). This rate did not differ significantly between control and intervention sectors.

In the post-intervention period, a total of 103 people were infected in control areas, and only 2 in the intervention sectors, giving incidence rates of $5.1 \%$ in the control sectors and $0.1 \%$ in the intervention sectors (Table 1). Thus the incidence of CL in the intervention areas decreased by about $97 \%$ while it increased by approximately $6.3 \%$ in the control areas. The decline in CL incidence in the intervention group for the year 2004-2005 was statistically significant $(P<0.05)$. The relative risk using Olyset ITNs in the intervention districts of Shiraz compared to the control areas was $0.02(P<0.05)$ and the efficacy was 0.98 . Accordingly, Olyset ITNs prevented $98 \%$ of cases of CL in the intervention areas (Table 2).

The 2 cases in the intervention areas were both men, aged 27 and 39 years. The most highly infected age group was 21-30 years and the lowest rate was in the age group 50 years. The Fisher exact test showed no statistically significant difference in the prevalence of ulcers by sex.

\section{Entomological results}

The total number of sandflies collected in the study areas between July 2004 and June 2005 was 2305 in Sedeh (1497 indoors and 807 outdoors) and 8711 (5031 indoors and 3680 outdoors) in Shiraz. The following 4 species were found in Sedeh: P. sergenti (53.0\%), P.papatasi (27.8\%), P. caucasicus $(0.8 \%)$ and Sergentomyia sintoni (18.4\%). In Shiraz we found: P. sergenti $(51.9 \%)$, $P$. papatasi $(18.0 \%), P$. major $(4.2 \%), P$.

\begin{tabular}{|c|c|c|c|c|c|}
\hline \multirow[t]{2}{*}{ Sector } & \multicolumn{2}{|c|}{ No. of people with } & \multirow{2}{*}{$\begin{array}{l}\text { Total no. } \\
\text { of people } \\
\text { exposed }\end{array}$} & \multirow{2}{*}{$\begin{array}{c}\text { RR } \\
(95 \% \mathrm{Cl})\end{array}$} & \multirow{2}{*}{$\begin{array}{c}\text { Efficacy } \\
(\%)\end{array}$} \\
\hline & CL & No CL & & & \\
\hline Intervention & 2 & 3810 & 3812 & 0.02 & 98 \\
\hline Control & 117 & 3698 & 3815 & $(0.00-0.07)^{*}$ & \\
\hline
\end{tabular}

${ }^{\star} P<0.05$.

$R R=$ relative risk; $\mathrm{Cl}=$ confidence interval. 
wenyoni $(0.3 \%), P$. alexandri (1.6\%), $P$. simici $(0.1 \%)$, S. sintoni $(17.9 \%)$, S. dentata (2.7\%), S. theodori (1.6\%), S. pawlowski $(0.9 \%)$ and $S$. clydei $(0.7 \%)$.

$P$. sergenti, the major vector of $L$. tropica, was the most abundant species, comprising $50 \%$ of the total number of sandflies caught. Figure 2 shows the seasonal population fluctuations of female $P$. sergenti captured in Sedeh and Shiraz during the survey period. There were statistically significant differences in the monthly catches of $P$. sergenti between control and intervention sectors in both cities $(P<0.05)$. The total number of female $P$. sergenti captured indoors in the control sectors was greater than inthe intervention sectors. There were 2 peaks, in July and in September, in the intervention and control areas. In both areas the highest population was recorded in August (Figure 2). In both cities, dead sandflies were found in the morning around the Olyset ITNs during survey team visits to households in the intervention sectors.

\section{Discussion}

Long-lasting ITNs are an efficacious method for the control of anthroponotic CL in endemic areas. Field trials with ITNs to prevent $C L$ have been undertaken in Afghanistan, Syrian Arab Republic and Turkey $[6,12,13]$. Our study is the first to explore the effectiveness of Olyset long-lasting permethrin-impregnated ITNs for the control of anthroponotic CL in the Islamic Republic of Iran. It showed the use of Olyset ITNs can provide significant personal protection from the bites of sandflies and subsequently reduce the risk of $C L$ infection.

Although there was a decrease in CL infection in Sedeh control sectors after the intervention, the number of new cases was not reduced to zero as it was in the intervention areas. In Shiraz, in contrast, there was an increase in the number of cases in control sectors and a large decrease in intervention areas. The efficacy of ITNs observed in this study confirms the findings of another trial in Bam, Islamic Republic of Iran [14].
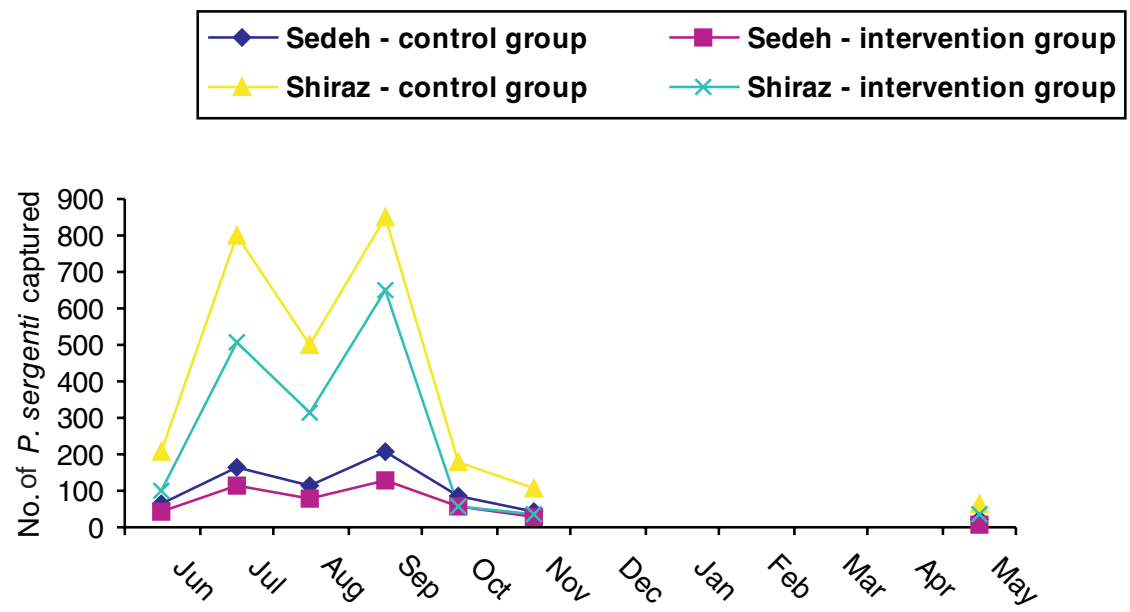

Figure 2 Seasonal fluctuations of number of female Phlebotomus sergenti captured in the control and intervention areas of Sedeh and Shiraz cities from June 2004 to May 2005

المجلة الصحية لشرق المتوسط، منظمة الصحة العالمية، المجلد الخامس عشر، العدد 0، 9.ب 
However, their results showed higher CL incidence among women in the intervention area. This was not the case in our study: we found no difference in incidence among men and women in the intervention areas compared with the control areas. These results were confirmed by studies carried out in the Syrian Arab Republic [6] and Turkey [13].

It seems that people using Olyset ITNs were still exposed to some sandfly biting activity, apparently due to sandflies penetrating the mesh of the bednets or possibly due to lack of compliance. An infected sandfly entering the net may still transmit the infection (and die afterwards), but the fact that not a single case was recorded in Sedeh intervention areas refutes such an assumption. Because bednets with finer mesh size may be unpleasant to use in the hot weather, Olyset ITNs $\left(50\right.$ holes/inch $\left.{ }^{2}\right)$ are the most practical way to maximize their protective efficacy.

ITNs have also been employed successfully against Anopheles mosquitoes and their recent use in wide-scale control programmes has resulted in a significant reduction of malaria morbidity and mortality $[4,9]$.

For sandflies, deltamethrin-impregnated bednets provided significant reduction in the biting rates of Lutzomyia youngi in Colombia [15] as well as $P$. sergenti and $P$. papatasi in the Syrian Arab Republic [6] and Turkey [13]. Similarly bednets and curtains impregnated with permethrin $100 \mathrm{mg}$ a.i. $/ \mathrm{m}^{2}$ provided significant reduction in the biting activity of $P$. papatasi in Khartoum, Sudan [16].

It is interesting to note that Sedeh was a new focus of anthroponotic CL and the promising results of this study may allow large-scale field trials in an epidemiologically similar area where there are new foci of CL. Altogether the use of Olyset ITNs provided a significant protection against CL to the populations at risk and with an efficacy of $98 \%$. The longitudinal design of the study allowed all individuals to be followed up during the study period (July 2004-July 2005 ) every month after the distribution of the ITNs (intervention period). This helped reduce the margin of error and made the estimate of previously infected individuals more accurate. The intervention and control sectors in both cities were similar in character and the differences in infection rates could be attributed to the intervention. The 2 men infected in the intervention sectors were infected at the beginning of the trial (August).

In summary, we believe that the use of Olyset ITNs provides personal protection in reducing the outdoor man-biting rate of $P$. sergenti and furthermore that the widespread use of nets, as shown in this trial, strongly confers some mass effect by reducing sandfly numbers. Use of Olyset ITNs would provide an invaluable control measure against these vectors of anthroponotic CL and may have good results with regard to the interruption of transmission of CL.

\section{Acknowledgements}

This investigation received technical and financial support from the WHO Eastern Mediterranean Region (EMR), Division of Communicable Diseases (DCD) and the WHO Special Programme for Research and Training in Tropical Diseases (TDR): EMRO/DCD/TDR Small Grants Scheme for Operational Research in Tropical and Communicable Diseases. Our thanks are due to Dr Luigi Gradoni for his valuable direction. 


\section{References}

1. Desjeux $P$. The increase in risk factors for leishmaniasis worldwide. Transactions of the Royal Society of Tropical Medicine and Hygiene, 2001, 95:239-43.

2. Nadim A, Javadian E, Mohebali M. The experience of leishmanization in the Islamic Republic of Iran. Eastern Mediterranean health journal, 1997, 3(2):284-9.

3. Korenromp EL et al. Monitoring mosquito net coverage for malaria control in Africa: possession vs. use by children under 5 years. Tropical medicine and international health, 2003, 8(8):693-703.

4. Ter Kuile FO et al. Impact of permethrintreated bed nets on malaria and all-cause morbidity in young children in an area of intense perennial malaria transmission in western Kenya: cross-sectional survey. American journal of tropical medicine and hygiene, 2003, 68(4 Suppl.):100-7.

5. Bern $\mathrm{C}$ et al. Factors associated with visceral leishmaniasis in Nepal: bed-net use is strongly protective. American journal of tropical medicine and hygiene, 2000, 63:184-8.

6. Tayeh A, Jalouk L, Al-Khiami AM. A cutaneous leishmaniasis control trial using pyrethroid-impregnated bednets in villages near Aleppo, Syria. Geneva, World Health Organization, 1997 (WHO/ LEISH/97.41).

7. Ashford DA et al. Studies on control of visceral leishmaniasis: impact of dog control on canine and human visceral leishmaniasis in Jacobina, Bahia, Brazil. American journal of tropical medicine and hygiene, 1998, 59:53-7.

8. Guillet PF. Long lasting insecticidal nets: current status and programmatic issues. Geneva, World Health Organization, 2004 (http://www.malariaconsortium.org/data/ files/pages/llin_7.pdf, accessed 23 April 2009).
9. N'Guessan R et al. Olyset Net efficacy against pyrethroid-resistant Anopheles gambiae and Culex quinquefasciatus after 3 years' field use in Cote d'Ivoire. Medical and veterinary entomology, 2001, 15:97-104.

10. Motavalli-Emami M, Nilforoushzadeh M. A new focus of cutaneous leishmaniasis due to Leishmania tropica in the central of Iran. Paper presented at the Third Congress of Leishmaniasis, Palermo, Italy, 10-15 April 2005.

11. Mesghali TO. On the Phlebotominae of Iran. Journal of medical entomology, 1964, 1:285-300.

12. Reyburn $\mathrm{H}$ et al. A randomized controlled trial of insecticide-treated bednets and chaddars or top sheets, and residual spraying of interior rooms for the prevention of cutaneous leishmaniasis in Kabul, Afghanistan. Transactions of the Royal Society of Tropical Medicine and Hygiene, 2000, 94:361-6.

13. Alten $B$ et al. Evaluation of protective efficacy of K-OTAB impregnated bednets for cutaneous leishmaniasis control in Southeast Anatolia-Turkey. Journal of vector ecology, 2003, 4:53-64.

14. Nadim A et al. Evaluation of pyrethroid impregnated bednets for control of anthroponotic cutaneous leishmaniasis in Bam (Islamic Republic of Iran). Geneva, World Health Organization, 1995 (WHO/ LEISH/95.37).

15. Alexander B et al. Evaluation of deltamethrin-impregnated bednets and curtains against phlebotomine sandflies in Valle del Cauca, Colombia. Medical and veterinary entomology, 1995, 9:279-83.

16. Elnaiem DA et al. Impact of pyrethroidimpregnated curtains on Phlebotomus papatasi sandflies indoors at Khartoum, Sudan. Medical and veterinary entomology, 1999, 13:191-7. 